\title{
BMJ Open Physician user satisfaction with an electronic medical records system in primary healthcare centres in Al Ain: a qualitative study
}

\author{
Shamma Al Alawi, ${ }^{1}$ Aysha Al Dhaheri, ${ }^{1}$ Durra Al Baloushi, ${ }^{1}$ Mouza Al Dhaheri, ${ }^{2}$ \\ Engela A M Prinsloo ${ }^{3}$
}

To cite: Al Alawi S, Al Dhaheri A, Al Baloushi D, et al. Physician user satisfaction with an electronic medical records system in primary healthcare centres in Al Ain: a qualitative study. BMJ Open 2014;4:e005569. doi:10.1136/bmjopen-2014005569

- Prepublication history and additional material is available. To view please visit the journal (http://dx.doi.org/ 10.1136/bmjopen-2014005569).

Received 26 April 2014 Revised 7 October 2014 Accepted 17 October 2014

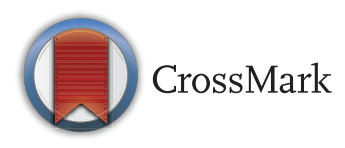

${ }^{1}$ Ambulatory Health Care Services, SEHA, Al Ain, Abu Dhabi, United Arab Emirates ${ }^{2}$ Homecare Department, Tawam Hospital in Affiliation with Johns Hopkins Medicine, Al Ain, Abu Dhabi, United Arab Emirates ${ }^{3}$ Department of Family Medicine, College of Medicine and Health Sciences UAE University, Al Ain, Abu Dhabi, United Arab Emirates

Correspondence to Dr Durra Al Baloushi; d_albaloushi@hotmail.com

\section{ABSTRACT}

Objectives: To explore physician satisfaction with an electronic medical records (EMR) system, to identify and explore the main limitations of the system and finally to submit recommendations to address these limitations.

Design: A descriptive qualitative study that entailed three focus group interviews was performed among physicians using open-ended questions. The interviews were audiotaped, documented and transcribed verbatim. The themes were explored and analysed in different categories.

Setting: The study was conducted in primary healthcare centres (PHC) in Al Ain, United Arab Emirates (UAE).

Participants: A total of 23 physicians, all using the same EMR system, attended one of three focus groups held in PHC in Al Ain Medical District. Each focus group consisted of 7-9 physicians working in $\mathrm{PHC}$ as family medicine specialists, residents or general practitioners.

Primary outcome measure: Physician satisfaction with the EMR system.

Results: Key themes emerged and were categorised as physician-dependent, patient-related and systemrelated factors. In general, physicians were satisfied with the EMR system in spite of initial difficulties with implementation. Most participants identified that the long time required to do the documentation affected their practice and patient communication. Many physicians expressed satisfaction with the orders and results of laboratory and radiology functions and they emphasised that this was the strongest point in the EMR. They were also satisfied with the electronic prescription function, stating that it reduced errors and saved time.

Conclusions: Physicians are satisfied with the EMR and have a positive perception regarding the application of the system. Several themes emerged during this study that need to be considered to enhance the EMR system. Further studies need to be conducted among other healthcare practitioners and patients to explore their attitude and perception about the EMR.

\section{Strengths and limitations of this study}

- The electronic medical records (EMR) system (Cerner) was introduced in the Emirate of Abu Dhabi, but only Al Ain clinics were selected for the study and, owing to the study design, the findings cannot be generalised.

- This is the first local study to address EMR user satisfaction adds a new user perspective.

- This study focused on the primary healthcare physician EMR users excluding hospital users and related healthcare professionals.

- Method of focus-group recruitment contributed to selection bias.

\section{INTRODUCTION}

The electronic medical record (EMR) is a new and promising tool for enhancing national and international healthcare delivery. ${ }^{1}$ Recent research has shown that information technologies can reduce medication errors, ${ }^{2}$ improve adherence to clinical practice guidelines, ${ }^{3}$ and improve the delivery of preventive health services, ${ }^{4}$ thereby potentially improving health outcomes for patients. ${ }^{5}{ }^{6}$ While electronic medical users can be productive, any disparities in experience, understanding and skills can leave team members feeling less than satisfied and not working to their full potential. ${ }^{1}$ Clinicians' perception of the EMR is a crucial determinant of the successful use of the EMR system. United Arab Emirate, Health Authority of Abu-Dhabi (HAAD) has implemented a system developed by one of the top three Healthcare IT vendors in the USA. $^{6}$ They have been in existence since 1979 and have installations in many countries including the USA, Canada, Australia, Saudi Arabia, Qatar, UAE, France, Spain, Singapore, Malaysia and South America. 
UAE has implemented the EMR system (Cerner) in 2008 in Abu Dhabi and Al Ain. Information and research studies related to user satisfaction is lacking in the local context.

This research study focused on physician user satisfaction with the EMR system in primary healthcare centres (PHC) in $\mathrm{Al}$ Ain and was the first known survey conducted in the UAE exploring this research question.

The findings of the quantitative study are reported in separate paper. We conducted a concurrent qualitative study in the same practices selected for the quantitative project.

The use of focus group interviews is becoming increasingly popular in healthcare research to explore the beliefs, feelings, attitudes and behaviour of individuals. Focus group discussions provide information about a range of ideas and feelings of individuals about specific issues and it illuminates the differences in perspective between groups of individuals. A focus group can generate a large amount of data in a relatively short time span. $^{7}$

In this study, the researchers explored user knowledge, attitude and satisfaction with the EMR system in PHC in $\mathrm{Al}$ Ain.

\section{METHOD}

Study design

This descriptive qualitative study was conducted in parallel with a quantitative study reported separately in a paper presented at the 2nd Al Ain Family Medicine Research Day on 3 March 2012 at Al Ain, UAE.

\section{Study method}

A purposive sampling strategy was used to recruit the physicians. ${ }^{8}$ The study was conducted in English. Permission was obtained from the clinic supervisors of each hospital prior to the study. Invitation letters were distributed among the physicians in clinics where the quantitative study on the EMR system was conducted. The management personnel were requested to select the participants for our study. These workers were selected based on their willingness to share their experiences on the EMR with us. Those who were to participate in the qualitative study were contacted by telephone 1-2 days before the focus group meeting. The physicians were not compensated for their time since most of them were released during their shift hours. The authors contributed to different aspects of the research study. The third author, a family medicine resident, reviewed the literature related to qualitative research, received additional training related to qualitative research methods, developed the moderators guide ${ }^{8}$ and moderated the focus groups. The three other researchers were respectively responsible for audiotaping and documenting verbal and non-verbal responses. Participants signed a consent form before the focus group session. All focus group interviews were conducted in the same PHC. To maximise ease of participation, the interviews were held after office hours during lunchtime. We deliberately exempted the managerial representation from our focus groups. The main reason was that we were of the opinion that their presence would cause junior colleagues to feel uncomfortable and prevent them from sharing their personal experiences and perceptions on their use of the EMR in the workplace.

The moderator introduced herself at the beginning of the focus groups, explaining the purpose of the study and assuring confidentiality of the information shared. ${ }^{8}$ The facilitator encouraged participation of all members in the discussions using open-ended questions and prompts focusing on the: (1) initial impression about the EMR system, (2) advantages and disadvantages of the EMR, (3) patients' reaction to the introduction of the EMR and (4) suggestions to improve the EMR. Interview questions were reviewed as the study progressed to seek further clarifications. ${ }^{9}$ See the online supplementary appendix A for detailed focus group questions.

Focus group interviews were conducted on three consecutive days. Each focus group interview lasted for an hour. Theme saturation was approximately achieved during the second focus group interview, and a third focus group interview was conducted to confirm the saturation.

\section{Data analysis}

The interviews were audiotaped and transcribed verbatim. As the interviews progressed, data were analysed after each focus group interview to develop preliminary codes to identify important and new ideas emerging. Each transcript was independently reviewed and coded separately by all the researchers to establish the main concepts. ${ }^{1}$ Subsequently, each transcript was analysed by each investigator independently to explore the themes and subthemes and then reviewed by the other investigators to compare and group the similar data. Further relations and triangulations ${ }^{10}$ were analysed during regular meetings. The next stage involved identifying the theme frame using the 'Krueger' framework. ${ }^{11}$ Trustworthiness of the data was enhanced by using Guba's four criteria. ${ }^{12}{ }^{13}$ For more details see online supplementary appendix B.

\section{Findings}

A total of 23 physicians attended one of the three focus groups. The overall focus group attendance was $70-80 \%$. The main reason given for non-participation was lack of sufficient time. Each focus group consisted of 7-9 physicians working in the PHC as family medicine specialists, residents or general practitioners using the same EMR system since 2008. The characteristics of the focus group participants are reported in table 1.

Each focus group consisted of a mix of males and females of different age groups and professional experience. 


\begin{tabular}{|c|c|c|c|}
\hline Demographic data & FG1 $(n=7)$ & FG2 $(n=9)$ & FG3 $(n=7)$ \\
\hline \multicolumn{4}{|l|}{ Gender } \\
\hline Male (female) & $3(4)$ & $4(5)$ & $2(5)$ \\
\hline \multicolumn{4}{|c|}{ Professional experience } \\
\hline Seniors & 5 & 6 & 4 \\
\hline Juniors (residents) & 2 & 3 & 3 \\
\hline \multicolumn{4}{|l|}{ Nationality } \\
\hline UAE & 2 & 3 & 3 \\
\hline Non-UAE & 5 & 6 & 4 \\
\hline
\end{tabular}

Several themes emerged from the focus groups about the implementation of the EMR (table 2). The main themes were categorised as physician issues, patient issues and system (Cerner) issues. These categories of main themes were arrived at through consensus during analysis of the focus group transcripts after the interviews. Participants repeatedly referred to or mentioned these themes during their discussions.

\section{Physician-dependent factors}

The initial impression of physicians

In general, physicians spoke favourably about the EMR system implementation, for example, "I think that, I do believe that my first impression was so amazing" (FG1), but all remarked that the beginning was difficult, for example, "At the beginning, as anything when you use it for the first time, it will look complex until you get familiar to the system" (FG3).

\section{Computer skills}

They believed that computer skills had a major role in understanding the EMR as they mentioned that old generation physicians were slower in typing and learning new tricks. There is a difference in competency among physicians in dealing with technology, for example, "Old generation doctors, whom I respect a lot of course, let's say there is a urine culture results, they don't know that there is a click where you can get the susceptibility" (FG1). Another example, "if you don't know like Alt and $\mathrm{C}$ is copying and Alt and V is pasting, (it takes) for a lot of people it causes a lot of difficulties" (FG2).

"For me for example if I want to explain something for the patient in anatomy, instead of drawing I will just enter the Google and the patient will be very happy: ohm, this is how it look, this is how the anatomy. And when you want to illustrate the disease process through pictures the patient will be very happy." It was also useful to provide the patient with very useful educational materials.

\section{The training}

Physicians appeared to have various opinions about the training period. Some were completely satisfied, for example, "It was sufficient, the training was good, of course the training itself to how to deal with computer at the beginning start in a good way" (FG3), while others were not satisfied and expressed that they were not aware of some facilities available in the EMR system, for example, "How to order everything at the start was very clear and comprehensive in the training part but when we start on the note part the training was not sufficient, in my opinion" (FG3). Some physicians suggested having individualised training sessions according to the physician needs. "I think they should work on teaching session, according to level of each, e.g. dividing them in groups and take them step by step even if it take 10 sessions or more" (FG2).

Participants specified that the IT team and super users were always available during the early time of implementation. They also suggested having regular meetings with the IT team to re-evaluate the physicians, answer their queries and have an updated training session for each system upgrade, for example, "they make a training they have to meet the users again to evaluate them. For example, I am using the Cerner and I collect questions there should be someone professional to answer me" (FG3).

"They should give us updating; now what I learn 2 years ago I am developing myself. This should be like regular because this will answer a lot of questions for me for the system" (FG1).

\section{Patient-related outcomes \\ Patient-physician relationship}

Physicians' perceptions about patient reaction were mixed. Initially, they were unhappy because of the disturbed patient-doctors relationship, for example, "It was bad but now it is improving a lot" (FG1) and "The real thing is eye contact is missing" (FG2). Furthermore, the waiting time increased due to data entry causing more frustration to the patients, for example, "The patient upset because of waiting time" (FG3).

Physicians believed that the waiting time was not caused by them but was mainly in the registration and nursing assessment, for example, "I found that nursing assessment they have to do a lot of things" (FG2). However, they believed that the benefits outweighed the waiting time issue and included beneficial issues as improved patient care, patient education and the health maintenance schedule. They stated that the patient flow was initially reduced but eventually returned to the same level as prior to implementation of the EMR, for example, "the same, the same" (FG2).

Many physicians were concerned about their patients' perception about the new technology. They felt that many patients were unhappy but indicated that few patients approved and made positive remarks to their physicians.

Physicians tried to adapt some strategies to maintain their relationship with their patients. Some were talking to their patients while dealing with the computer so that the patients would not feel neglected, for example, "ok now I am checking your results, I am checking your past file" (FG1).

Others reserved data entry work for immediately after the visit, for example, "we can put the diagnosis, then 
Table 2 Summary of themes of all focus groups

\section{Themes and subthemes}

\section{Quotes}

Physicians-dependent 1 . The initial impression about the EMR system factors

- Difficulty in use at the beginning

"Still we are in the fetal state." FG1"We had a team

- Training was sufficient and good

2. Past computer skills

which was always available." FG3

- Different users' generations with different computer skills

"Old generation doctors, whom I respect a lot of course, let's say there is a urine culture results, they don't know that there is a click where you can get the susceptibility." FG1

3. The impression about the precompleted "Definitely, it saves a lot of time." FG2 notes

- Precompleted notes definitely saves time

Patient related

4. Doctor-patient relationship

- No eye contact

- Waiting time is more

- Patients are accepting the system because it is reflecting an advance modern of technology

System-dependent factors

5. Complexity of the system

- The EMR complexity was at the beginning

- Complexity of the system, not specialised to $\mathrm{PHC}$

6. The quality of documentation

- Documentation now is readable and better than handwriting

- The quality of documentation is depends on the physician themself

7. The process of prescription in the Cerner and the current problems

- Prescription is better and safe now

- Allergy system decreasing the medication errors

8. Improvement of the orders and results with the EMR

- The orders and the result much organised

- Fast feedback of the results

9. Referral issues with the Cerner

- Referral issue easy with feedback

- Trace patient's appointment and print it for them

10. Confidentiality

- No confidentiality with the EMR

11. Disadvantages of the EMR

- Takes time

- Important notes should be highlighted

12. Suggestions to improve the EMR

- Giving more time

- Meetings and updating by Cerner people
"Initially the patient were not happy." FG1

"No eye contact." FG1

"It consumes more time." FG1

"Patient will accept this new system because it is more advance and reflect that the clinic is more advance with modern technology but giving good care." FG1

"If you get use to it, yes, it become very easy." FG1

"The system was not designed for primary care." FG3

"Before we should open this charts. I can't read handwriting of the doctors, now everything is easy and everything is in front of my eyes only by

clicking." FG2

"Definitely much better 100\%." FG1

"Before there were so many mistakes." FG2

"If there is allergy, decrease the error because

during hand writing there was medication errors."

FG1

"The stronger point on cerner is lab's and xrays."

FG3

"Much organized." FG1

"The results will come directly to your inbox." FG1

"Before when was referring patients to the hospital we don't have any clue what happened to him." FG3

"I can easily open the system and look for it and tell her this is your appointment." FG1

"It is easy to break this confidentiality with the cerner. Any body can open the file." FG1

"Longer, even not only with doctor, from pharmacy side, from reception side." FG3

"It is difficult to eye scan, it should be highlighted."

FG1

"Give us enough time." FG1

"They should give us updating; now what I learn 2 yrs. ago I am developing myself." FG1

EMR, electronic medical records; FG, focus group; PHC, primary healthcare centres.

put the medication, because we can't put medication without diagnosis then put the labs then ask the patient to go and continue documentation" (FG2).
"The proper thing is to take full history from the patient, maintaining the good communication with the patient then turn and document" (FG3). 
All physicians believed that the presence of the EMR had strong effects on the flow of the patients initially, but later returned to the prior situation.

Some of the physicians used the electronic health records as a means for collaboration to share the screen with their patients. They showed them some pictures to illustrate and explain concerns.

\section{System-dependent factors}

A summary of the advantages and barriers highlighted by physicians using the EMR is discussed in the text below.

\section{The quality of documentation}

Physicians believed that the EMR improved the quality and clarity of the documentation, for example, "it is very helpful, very readable, better than the handwriting"; another example, "previously they were usually write their own abbreviations 'LE', 'RE' not sure what they mean is it LEFT EYE or the disease itself but now because of the system coding they tend to write" (FG2). However, some physicians described the system as complex and less informative, for example, "if the doctor is free texting he will say the real thing and when you read it you will know what is the meaning exactly (overlapping talk) but if you tick tick, tick sometime you lose" (FG3).

Participants in all focus groups agreed that the current EMR was designed mainly for the hospitals and not for the primary care centres, for example, "The system was not designed for primary care (all agree) it is designed for hospitals this is the main issue for us" (FG3). Physicians had difficulties finding a diagnosis for some of the common conditions like skin laceration or skin abrasion seen in daily practices.

\section{System complexity and interconnectivity}

A common theme was the complexity of the system. Participants explained that they had difficulty at the beginning of implementation of the system to find the proper coding for the diagnosis. They also reported that sometimes they had to duplicate and repeat notes in several locations because there was no link, for example, between the notification system and the patient notes, for example, "Notification system, there must be a connection between Health Authority Abu Dhabi and cerner (EMR) another thing some cases...if anyone experience how to notify a case of syphilis he will hate himself (laughing). Four pages you must fulfill four (4) pages" (FG3).

Participants were very satisfied with the pre-completed notes in the system. They mentioned that it helped them save time and was very useful in the specialty clinics, for example, "Definitely, it saves a lot of time" (FG1); another example, "Helpful, especially in the clinics, the specialized clinics like the well-baby clinic, in antenatal clinic, in chronic clinic" (FG1). They also emphasised that in the long run the review of accumulated documentation will be challenging by asserting that visual scanning is impossible without highlights, for example, "Accumulation over the year will be a problem because you cannot go through all the note to find something" (FG1).

\section{Ordering and viewing}

Many participants in the three focus groups were very pleased and satisfied with the orders and results of laboratory and radiology functions. They mentioned that it is the strongest part in the EMR system and the results are available on the same day, for example, "The stronger point on cerner (EMR) is lab's and x-rays" (FG3). Participants found that online orders from the Cerner tick list were easier than the written ones, for example, "If you are comparing writing an order with ticking order, ticking order is easier" (FG3).

The EMR viewing capability was considered to be useful information for patient management because it helped with continuity of care and to follow progression of many chronic diseases, for example, "For example, if you have a patient with renal failure you can see the results (creatinine) for one year which is very useful" (FG2).

Participants believed that X-ray orders are very helpful because the radiologist has access to the history of the patient, for example, "It was really miserable because there is no history for the doctor to read from x-ray. When I sit with the doctor the radiologist, I feel what he is feeling because there is nothing just X-ray. Okay for what? What are you thinking? What are your differential, it is nothing" (FG3).

Regarding the electronic prescription, participants were very excited since it helps in reducing the errors. "It is easy and safe also" (FG1). They indicated that the prescription refill system saved time. Participants stated that they liked the drug reference text that appeared with each medication order.

Participants suggested agreeing on uniformity in the use of metric units deciding on either reporting in milligram or millimol. Several participants agreed that the EMR referral is much easier and patients could be traced and followed up through the system. Feedback about patient referral and management was a major improvement according to participants. The previous paper system did not support continuity of care or feedback, for example, "Before we don't know any feedback about the patient but now I refer one patient suspecting bronchiolitis or something after one hour I can open the cerner (EMR) and I can see what they did for him" (FG1).

According to some participants, the referral and feedback system enhances continuity of care of the patients; it provides them with a complete picture of post referral management and progress, for example, "I think referred for us as Family medicine for continuity of case is better" (FG2). Regarding the disadvantages of the EMR, participants reported that the system was time-consuming and required too much detailed documentation, for example, "Previously documentation was not such detail when 
using file. But whatever time we spent, we spent with patient, we were asking his history, examining, and writing a prescription giving him cause and the rest come but now, suppose URTI case come one or two minutes is taken to diagnose the case once the diagnosis is finished then I started with my computer so this computer is taking time and patient finished and he is just waiting and waiting till I finished so he gets upset" (FG1).

An important issue that was raised in the first focus group, and subsequently added to the discussion questions, was that of confidentiality. All participants agreed that there was no confidentiality with the EMR system, for example, "One of the main issues with the Cerner (EMR) is the confidentiality" (FG1).

\section{Suggestions}

One of the emerging themes from the discussions was a suggestion to improve the EMR system. Participants suggested the allowance of more time for the physicians and also that the email system be improved. They also proposed including some diagnoses in the EMR that are commonly used in the primary care setting, for example, "Common medical problem should be included in the diagnosis and encounter pathway should include more general complaints" (FG1).

In the second focus group, participants suggested that the electronic document design should be simplified for use by doctors and patients in primary care.

"Electronic documentation it is so much better. No one differ about that but it must be simplified for the patient and for the physician" (FG2).

Participants also suggested that allergies, problem list and diagnosis should be included in the main page to simplify the system. Physicians wanted to have a free text to add diagnosis and not be restricted to the available EMR list. For example, "We can't find $\mathrm{ICD}_{9}$ since one or two months it can enter as free text, now it can't I should change it. It should be applicable for change it. He was osteopenic and now osteoporosis. So I can change it I can click this and write other" (FG3).

Participants made a request for having a link between HAAD records and the EMR system for sick leave notes and notification of disease, for example, "Sick leave and notification. There must be a link between Cerner (EMR) and HAAD at HAAD website. For sick leave it is very important as we write free text and patient coming to me and take it after 3 days go to another clinic and take another sick leave like this" (FG2).

\section{DISCUSSION}

This is the first published paper in the UAE to evaluate the EMR users' satisfaction since the implementation. The aim of this study was to understand the attitude and knowledge of physicians about the EMR. Another goal was to identify the disadvantages and suggestions to improve the system.
The physicians' perceptions about the EMR summarised in the preceding text suggested several ideas to improve the system. Physicians in all focus groups were satisfied with the EMR system, although some physicians were facing some difficulties at the beginning of implementation. Most of the participants identified the long time required to do the documentation in the system as a factor that affects their practice and communication with the patients. The same results were found in a study conducted in Hawaii. Participants reported that the Clinical Information System had reduced clinicians' productivity, primarily because of extra work such as processing laboratory result reports, entering orders and navigating through the systems. ${ }^{14}$

Many physicians were pleased about the orders and results of laboratory and radiology as they emphasised that this is the strongest point in the EMR system. They were also happy about the electronic prescription because it reduced errors and saved time. In a survey conducted by Robert $e t a l,{ }^{15}$ including 2719 family physicians in the USA, the respondents highlighted the advantages of the EMR which were almost similar to our findings. Their respondents stated that they were pleased with the EMR system since it was fast, easy to use, well documented, more precise and provided patient engagement tools such as the patient education resources and patients' portal. ${ }^{15}$ However, American College of Physicians (ACP) and American EHR Partners conducted a survey reporting that physician dissatisfaction with the EMRs increased from 24\% in 2010 to $39 \%$ in 2012 . The reasons provided by the respondents for their dissatisfaction with the EMR was that the system was expensive and was not significantly reducing their workload. ${ }^{16}$

They mentioned that computer skills had a major effect on understanding the EMR. In the literature review, computer literacy was identified as a major barrier to the implementation of the EMR.

There was a finding that only emerged in the second focus group due to the presence of a physician who was previously exposed to the auditing process. The researchers were of the impression that physicians perceived the EMR as a significant threat when it was used to audit the physicians for documentation and patient confidentiality, for example, "the medical record do regular audit and find out, for example, why the chart has been opened".

Another example, "part of annual appraisal of the physicians is the (audio unclear) we have about eight competencies one of them is the documentation and we usually audit at least 10 to 20 task for each physician and all the important factors the presenting symptom, the history of present illness the past medical history... we do for audit and this is why the physician are keen to have a complete or as much as we can about full documentation." Physicians had a negative perception that they were monitored for their performance through the Cerner, which created some discomfort during the 
session. This finding was not commonly identified in our literature review except in one study where the respondent reported the feedback as personal criticism. ${ }^{15}$ It is important to ensure that during the implementation of a new system, like the Cerner, users should be informed about the purpose of the use of the system and also about the auditing tool and the purpose of use of audits to allay fears and negative perceptions.

The confidentiality issue was added to the moderators guide as a focus group questions after it emerged as a theme in the first focus group. Participants mentioned the loss of confidentiality in the patient's files, because anybody who has access could open any file. A new insight developed after the first focus group, and the interview questions were adapted to explore this new knowledge. It was discussed until the point reached saturation similar to the situation in other studies. ${ }^{17} 18$

Physicians in our study reported that the EMR documentation was time-consuming, due to many clicks that had to be performed, even for short documents and simple complaints. In the review of the published literature, physicians recognised the benefits of the EMR for legibility, and readily linked this to better and safer patient care outcomes. The burden and time inefficiency of data entry are seen as major disadvantages, suggesting the importance of 'smarter' and more intuitive data entry interfaces and perhaps voice recognition. ${ }^{19}$ This also emerged as a subtheme in our study.

Participants continued to identify the important role of an EMR champion within their practice who encouraged EMR usage and was available to solve problems. Support and encouragement from a 'champion' has been noted in the literature as crucial throughout the implementation process. ${ }^{1}{ }^{20}$ In this study, participants mentioned that follow-up by super users and the IT team would be beneficial.

Participants identified the messaging system within the EMR software as a practical, useful and important tool for enhancing efficiency within the team. Successful communication has been linked to increased patient safety and improved patient outcomes. ${ }^{1}$ The physicians in all focus groups emphasised this point. They mentioned that internal communication in the clinic through the system had saved time and improved patient safety.

Major barriers to implementation and adoption included computer literacy, training and time. There was also variability regarding the influence of prior computer knowledge on perceptions of EMR implementation. While these issues have been identified in prior studies, they remain an ongoing challenge for primary healthcare providers. Implementation and adoption of EMRs will be most successful when protected time is available to train all EMR users. ${ }^{17}$ In this study, similar concerns were raised.

A recent review of studies on barriers to EMR implementation found that these could be broadly categorised as concerns about costs, technical issues (including lack of interconnectivity, high complexity and lack of customisability), lack of time, psychological factors such as lack of belief in the EMR, social factors such as lack of support from colleagues, and legal issues such as concerns over privacy and security. ${ }^{21}{ }^{22}$ Complexity, interconnectivity and time factors also emerged from the current study.

\section{Limitations}

The present study was limited in several ways. First, it included only physicians despite the importance of understanding nurses, pharmacists and other healthcare professionals' beliefs about using the EMR. Second the study was conducted only in Al Ain district, although HAAD has implemented the EMR system in Abu Dhabi and $\mathrm{Al}$ Ain. This study focused on EMR users in primary healthcare settings and did not include EMR users in hospitals. The application of purposive sampling strategy in the recruitment of physicians during this study is also a limitation. Since the respondents were self-selected, it might mean that this study had many EMR enthusiasts.

\section{Conclusion}

Clinicians have a positive perception regarding the application of the EMR in the primary care outpatient practices. However, several themes emerged during this study that need to be considered to enhance the EMR system. Therefore, further studies need to be conducted by focusing on other medical users and patients in viewing their attitude and perception about the EMR system. Adapting the system to needs and diagnosis common to the PHC setting and offering continuous training and technical support would assist in convincing apprehensive EMR users.

\section{Recommendations}

A crucial subsequent step is selecting from the themes which emerged in the study, the themes that are most commonly mentioned or most important to physicians, and to formulate a hypothesis about the mechanisms by which these beliefs might shape acceptance and user behaviour. A follow-up survey should be implemented on nurses, pharmacists, patients and other groups so as to understand their beliefs and attitudes about the EMR system. The findings which correspond with those of other studies or refer to issues that have a negative impact on services and can be rectified should be communicated to authorities and IT vendors to seek solutions to improve and adjust future applications.

Acknowledgements The authors thank the study participants for their kind cooperation and time, all managers of clinics included in the study for their co-operation and support, and those involved in the process of our research. Thanks especially to Mrs Maria Cristina-Community Medicine Department and Dr Latifa Al Ketbi-Department of Family Medicine.

Contributors DAB was the moderator of the focus group. SAA was the principal investigator and the coordinator of the study. SAA and AAD contributed to the analysis, interpretation and preparation of the manuscripts with inputs from all authors. EAMP, DAB and MAD were involved in editing the article or revising it critically for important intellectual content. All authors 
contributed to the concept and design of the study, and have read and approved the final manuscript.

Funding This research received no specific grant from any funding agency in the public, commercial or not-for-profit sectors.

Competing interests None.

Patient consent Obtained.

Ethics approval The proposal for this study was approved by IRB of the AI Ain Medical District Human Research Ethics Committee, protocol No. S011-3. Permission was taken from governing hospitals of each clinic before starting the study.

Provenance and peer review Not commissioned; externally peer reviewed.

Data sharing statement Further study data are not to be shared due to patient data confidentiality when the study was undertaken. The quantitative study has unpublished data available from the corresponding author, while appendices $A$ and $B$ are available for data sharing. Further details of the study protocols can be requested from the corresponding author by emailing (d_albaloushi@hotmail.com).

Open Access This is an Open Access article distributed in accordance with the Creative Commons Attribution Non Commercial (CC BY-NC 4.0) license, which permits others to distribute, remix, adapt, build upon this work noncommercially, and license their derivative works on different terms, provided the original work is properly cited and the use is non-commercial. See: http:// creativecommons.org/licenses/by-nc/4.0/

\section{REFERENCES}

1. Denomme LB, Terry AL, Brown JB, et al. Primary health care teams' experience of electronic medical record use after adoption. Fam Med 2011;43:638-42.

2. Bates DW, Leape LL, Cullen DJ, et al. Effect of computerized physician order entry and a team intervention on prevention of serious medication errors. JAMA 1998;280:1311-16.

3. Shea S, DuMouchel W, Bahamonde L. A meta-analysis of 16 randomized controlled trials to evaluate computer-based clinical reminder systems for preventive care in the ambulatory setting. J Am Med Inform Assoc 1996;3:399-409.

4. Gill JM, Ewen E, Nsereko M. Impact of an electronic medical record on quality of care in a primary care office. Del Med J 2001;73:187-94.
5. Crosson JC, Stroebel C, Scott JG, et al. Implementing an electronic medical record in a family medicine practice: communication, decision making, and conflict. Ann Fam Med 2005;3:307-11.

6. McBride M. Ranking Top 10 Hospital EMR Vendors by Number of Installed Systems. 25 March 2011 [Internet]. http://www.darkdaily. com/ranking-top-10-hospital-emr-vendors-by-number-of-installedsystems-32511\#axzz1jnXZOuRt

7. Rabiee F. Focus-group interview and data analysis. Proc Nutr Soc 2007;63:655-60.

8. Morae. Example Focus Group Moderator Guide [Internet]. 2009. http://www.assets.techsmith.com

9. Burnard P. Writing a qualitative research report. Nurse Educ Today 2004:3:174-9.

10. Kelliher F. Interpretivism and the pursuit of research legitimisation: an integrated approach to single case design. J Bus Res 1998;3:123-32.

11. Krueger R. Designing and conducting focus group interviews. Environment 2002;1-18.

12. Shenton AK. Strategies for ensuring trustworthiness in qualitative research projects. Educ Info 2004;22:63-76.

13. Pope $\mathrm{C}$, Ziebland S, Mays N. Analysing qualitative data. BMJ 2000;320:114-16

14. Scott JT. Kaiser Permanente's experience of implementing an electronic medical record: a qualitative study. BMJ 2005;331:1313-16

15. Robert L, Kenneth G, Adler M. The 2011 EHR user satisfaction survey: responses from 2,719 family physicians. Fam Pract Manag 2011;18:23-30.

16. ACP. Survey of clinicians: user satisfaction with electronic health records has decreased since 2010. American College of Physicians and American EHR Partners release survey results, 2013.

17. Terry AL, Giles G, Brown JB, et al. Adoption of electronic medical records in family practice: the providers' perspective. Fam Med 2009;41:508-12.

18. Wager KA, Lee FW, White AW, et al. Impact of an electronic medica record system on community-based primary care practices. J Am Board Fam Pract 2000;13:338-48.

19. Richard JH. Physicians' beliefs about using EMR and CPOE: in pursuit of a contextualized understanding of health IT use behavior. Int J Med Inform 2010;79:71-80.

20. Terry AL, Thorpe CF, Giles G, et al. Implementing electronic health records. Can Fam Physician 2008;54:730-6.

21. Greiver M, Barnsley J, Glazier $\mathrm{RH}$, et al. Implementation of electronic medical records. Can Fam Physician 2011;57:e390-7.

22. Boonstra A, Broekhuis M. Barriers to the acceptance of electronic medical records by physicians from systematic review to taxonomy and interventions. BMC Health Serv Res 2010;10:231. 